\title{
Development of a Stability Control Method for the Aero-Train*
}

\author{
Satoshi KIKUCHI ${ }^{* *}$, Fukuo OHTA ${ }^{* * *}$, Takuma KATO ${ }^{* * *}$, Tomomi ISHIKAWA ${ }^{* * * *}$ \\ and Yasuaki KOHAMA ${ }^{* * *}$ \\ **Gifu University, \\ 1-1, Yanagido, Gifu City, 501-1193, Japan \\ E-mail: kiikchi@gifu-u.ac.jp \\ ${ }^{* * *}$ Institute of Fluid Science, Tohoku University, \\ 2-1-1, Katahira, Aoba-ku,Sendai, 980-8577,Japan \\ E-mail: kohama@ifs.tohoku.ac.jp \\ ***AISIN AW Co., Ltd.,
}

\begin{abstract}
Taking into account the seriousness of the greenhouse effect, drastic measures must be taken to prevent carbon dioxide emissions from transportation systems. In Japan, over $20 \%$ of the carbon dioxide is emitted from transportation systems. Aero-Train is a new zero-emission high-speed vehicle proposed by the authors. Aero-Train levitates aerodynamically using the wing-in-ground effect (WIG). In order to investigate the stability of Aero-Train, wind tunnel experiments of the front body section of a test run model of Aero-Train (ART002) and a levitating test run of ART002 using an improved control program were performed. The results showed that the front section of the body is unstable or is weakly stable in the direction of yawing and rolling. In the levitating test run of ART002, levitated running without surface contact was achieved inside the guide way. The amplitude of the roll angle oscillation was reduced by more than $50 \%$ compared to that of the previous control program. However, the vibration of the rolling remained.
\end{abstract}

Key words: Motion Control, High Speed Ground Vehicle, Stability, Aero-Train, Wing-in-Ground Effect

\section{Introduction}

Aero-Train is a high-speed ground vehicle that levitates aerodynamically using the wing-in-ground effect (WIG). When a wing approaches the ground or a water surface, its lift-drag ratio increases greatly due to the air cushion effect under the wing. The presence of this effect has been known for quite a while. In the fields of both aerospace engineering and marine engineering, research and development of WIG vehicles over water surfaces has been conducted $^{(1)-(4)}$, with some of the development being done at the level of practical use. However, these WIG vehicles designed for water surfaces often have a large horizontal stabilizer in order to cancel the pitching instability, which is caused by changes in the center of wing pressure with levitation height. WIG vehicles for water surfaces have a high value of aerodynamic drag due to big hump drag and the inherent danger of making contact with the water. Therefore, this type of WIG vehicle is not yet accepted among the general public.

On the other hand, Kohama et al. proposed a transportation system that uses the wing-in-ground effect on land about 10 years ago $^{(5)}$. Called the GETS (Ground Effect 
Transport System), it is commonly referred to as "Aero-Train." Aero-Train runs along a track (guide way) where the circumference is almost completely covered (Fig. 1 and Fig. 2), and for this reason the Aero-Train has sufficient weather resistance and has neither a hump drag problem nor a danger of contacting the water. Moreover, Aero-Train can use the ground effect to maximum effect. Aero-Train uses a tandem wing arrangement that arranges two wings at both the front and rear of the body due to the shape-limitations of the guide way and the stabilizing effect in the pitching direction. The main wings have vertical side wings at their tips in order to automatically regulate its lateral position in the guide way by the wing-in-ground effect between the side wing and the side wall.

Since Kohama et al. proposed the Aero-Train, basic research required for its development has been conducted ${ }^{(5)-(11)}$. A summary of the research results of Kohama et al. is as follows.

1. When the trailing edge height is less than $10 \%$ chord, the ground effect works effectively, and the lift-to-drag ratio in this region rises 2 to 3 times compared to cases without the ground effect.

2. The most effective shape of the lower surface of the airfoil for the ground effect is a concave or a flat lower surface because of the high-pressure region that exists between the lower surface and the ground.

3. High stability in the pitching direction is difficult to maintain using a single wing. However, using a tandem wing arrangement with a long distance between the two wings, stability in the pitching direction is acquired.

4. When the distance between the front wing and the rear wing is more than 2.5 times the chord, it can prevent the performance deterioration of the rear wing.

5. When the angle of attack of the side wing is positive, repulsion from the side wall by the ground effect is generated. Moreover, the lift of the main wing increases as the side wing approaches the side wall.

6. A fillet in the junction part of the main wing and the side wings decreases the drag by wing-wing interaction effectively.

Concurrent to this research, test runs of the Aero-Train in the guide way at the Miyazaki Research Center, belonging to the juridical foundation "Railway Technical Research Institute (RTRI)," have been performed since 1999. Thus far, successful runs have been completed in the autonomic levitated test runs of two different models, the ART001 and ART002. The ART001 is a test prototype model for investigating the possibility of a high-speed transportation system using the ground effect. Because the possibility of such a high-speed transportation system using the ground effect was successfully proven from the experiment using the ART001, the purpose of the ART002 test became the speeding-up of the transport speed and further development of the control method. A photograph of the ART002 is shown in Fig. 2. Currently, it is only stable in the pitch when levitating. For this reason, it cannot be run without contacting the surface.

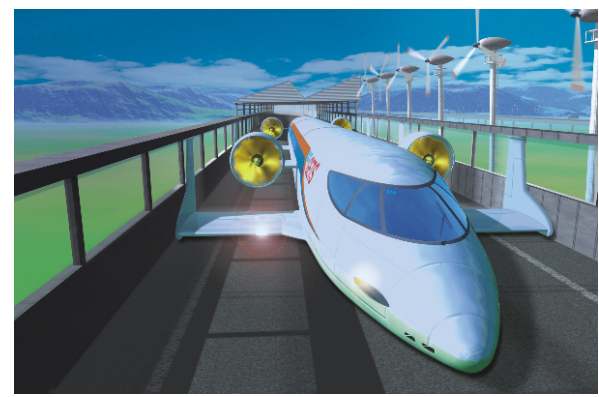

Fig.1 Conceptual design of Aero-Train

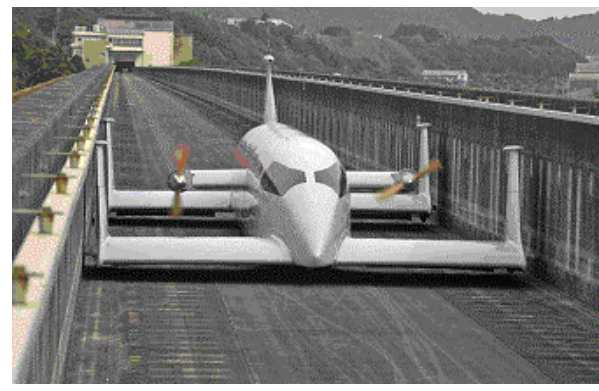

Fig.2 Experimental model ART002

In the current study, the two following tests 
were conducted to investigate the effects of the rolling angle, the yawing angle, and the lateral position on the stability of the Aero-Train.

1. The stability of the ART002 was evaluated by the aerodynamic measurement of the roll, yaw, and lateral position inside the guide way by a wind tunnel experiment of the body front section.

2. The levitated test runs were focused on the stabilization of the roll, yaw, and lateral position inside the guide way.

\section{Nomenclature}

$\begin{array}{llll}b & : & \text { span of the main wing, m } \\ c & : & \text { chord of the main wing, m } \\ C_{L} & : & \text { lift coefficient } & C_{L}=F_{L} /\left(0.5 \times \rho U^{2} S_{L}\right) \\ C_{R} & : & \text { rolling moment coefficient } \quad C_{R}=M_{R} /\left(0.5 \times \rho U^{2} S_{L} b\right) \\ C_{S} & : & \text { side force coefficient } & C_{S}=F_{S} /\left(0.5 \times \rho U^{2} S_{G}\right) \\ F_{L} & : & \text { lift, } \mathrm{N} \\ F_{S} & : & \text { side force, } \mathrm{N} \\ h & : & \text { levitation height, m } \\ M_{R} & : & \text { rolling moment around the center-of-gravity, } \mathrm{Nm} \\ M_{R}{ }^{\prime} & : & \text { rolling moment measured with a force transducer, Nm } \\ R e & : & \text { Reynolds number } \\ S_{L} & : & \text { area of the main wing, } \mathrm{m}^{2} \\ S_{G} & : & \text { area of the side wing, } \mathrm{m}^{2} \\ U & : & \text { freestream velocity or air speed, } \mathrm{m} / \mathrm{s} \\ \rho & : & \text { density of air, kg/m } \\ \theta & : & \text { pitching angle, deg } \\ \phi & : & \text { rolling angle, deg } \\ \psi & : & \text { yawing angle, deg }\end{array}$

\section{Stability Evaluation by Wind Tunnel Experiment}

\subsection{Wind Tunnel Facilities and Model}

The facility used in the experiments was the low-turbulence wind tunnel at the Institute of Fluid Science (IFS), Tohoku University ${ }^{(12)}$. An outline of the experimental apparatus is shown in Fig. 3. When the body tilts in the yawing direction, the side force (lift of the side wings) of the rear side wings stabilizes the body. However, the side force of the front side wings works in the opposite direction, making the body unstable. For this reason, the model used for the experiment comprised only the front section of the ART002. A wind tunnel experiment model was made at $1 / 10$ scale. The airfoil of the main wing and of the side wing was NACA6412 modified and ClarkY60, respectively. Both of the airfoils were the same shape as the airfoils of the ART002The mounting angle of the main wings was 2 degrees measured by the flat lower surface, and the mounting angle of the side wings was 0 degree. The guide way was imitated by installing two parallel aluminum plates (dummy side wall, 6 $\mathrm{mm}$ thick and $200 \mathrm{~mm}$ long) perpendicularly on an aluminum plate (dummy ground, $20 \mathrm{~mm}$ thick and $860 \mathrm{~mm}$ long). The distance between the two dummy side walls was set to 340 $\mathrm{mm}$, which is $1 / 10$ of the width of the guide way at the Miyazaki Research Center. The coordinate system was $X, Y$, and $Z$ in the streamwise, spanwise, and perpendicular directions, respectively. The model was connected to a 3-component force transducer (Nissho Electric Works Co., Ltd., LMC-3501-50N) by a support arm. The force transducer was fixed behind the model, and the model was supported from the back. The dummy body covered the support arm, and the connection of the dummy body and the model body had a 

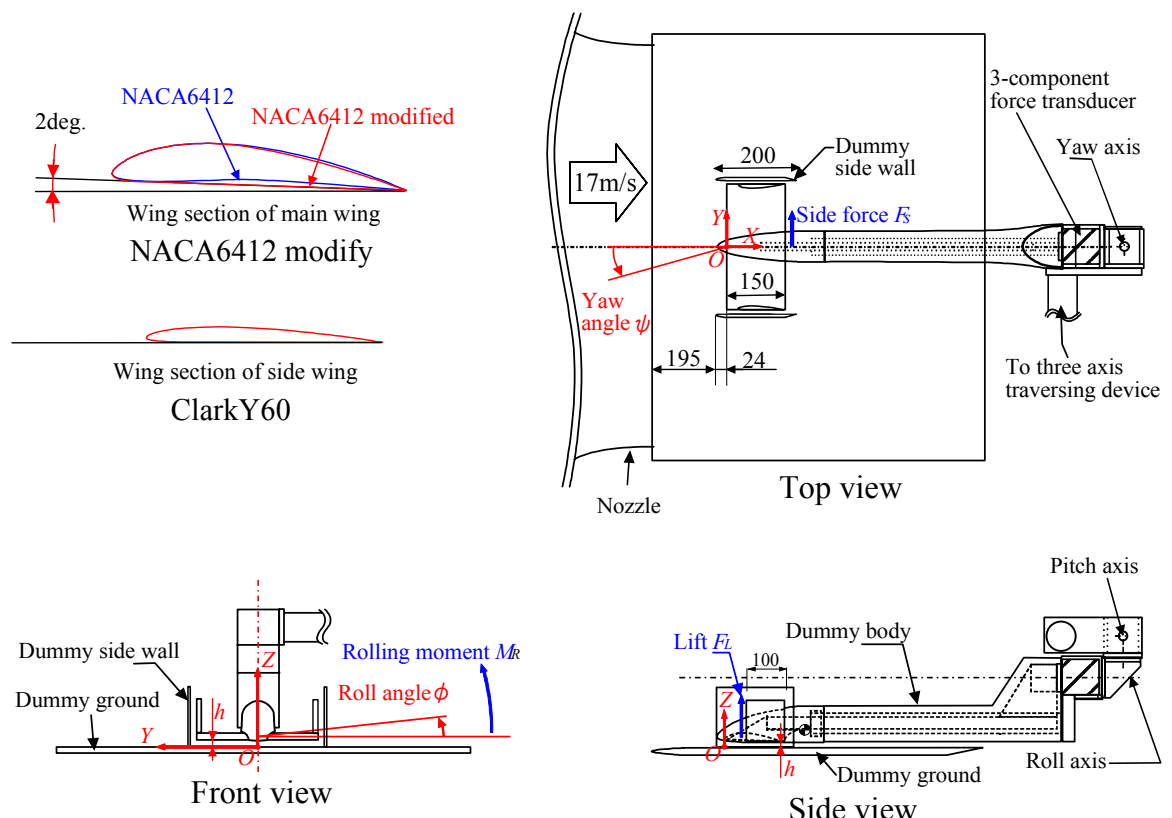

Side view

Fig.3 Experimental condition

simple labyrinth structure. For this reason, any force other than the force on the model was not detected. The 3-component force transducer was fixed to a 6-axis $(X, Y, Z$, pitching angle $\theta$, rolling angle $\phi$, yawing angle $\psi$ ) traverse system.

\subsection{Experimental Method}

In the experiments, the pitching angle $\theta$, the rolling angle $\phi$, the yawing angle $\psi$, the lateral position inside the simulated guide way $Y$, and the surfacing height $h$ are changed, while the lift $F_{L}$, the side force $F_{S}$, and the rolling moment $M_{R}{ }^{\prime}$ are measured by the 3-component force transducer. In this measurement system, the center-of-gravity of the body differs from the measurement position of the force transducer. For this reason, a rolling moment around the center-of-gravity $M_{R}$ is obtained by separating the effect of the lateral force $F_{S}$ from the measured rolling moment $M_{R}{ }^{\prime}$. It is not necessary to adjust the lift or the lateral force because both the lift and the lateral force acting on the model are detected directly. Wind velocity is fixed at $17 \mathrm{~m} / \mathrm{s}$ because of the restriction of the force transducer. Here, the Reynolds number is 175000 based on the main wing and 116000 based on the side wing. As shown in Fig. 3, upward lift $F_{L}$, rightward side force $F_{S}$, pitch in the pitch-up direction $\theta$, clockwise rolling moment $M_{R}$, clockwise rolling angle $\phi$, and clockwise yawing angle $\psi$ are all defined as positive.

\subsection{Results of Wind Tunnel Experiment and Discussion}

In order to achieve a levitated run in a guide way without contact on the walls, it is necessary that not only the height and lateral position of the body be stabilized by the ground effect of the main wings and side wings, but also that the angle changes (pitching angle, rolling angle, yawing angle) of the three axes are stabilized. The changes of the side force coefficient $C_{S}$, which is used as the index of stabilization of the transverse direction to the $Y$ position, when the rolling angles are 2 degrees, 0 degree, and -2 degrees, are shown in Fig. 4 (a) - (c). You can see that for any rolling angle $\phi$, the side force coefficient $C_{S}$ shows very little change with respect to $Y$, although it increases greatly in response to changes in yaw $\psi$. This means that there is almost no repulsion by the ground effect on the side wall, and that only the yawing instability of the front side wings exists. Therefore, the levitated run is unstable. This shift of the curve due to the change in yaw $\psi$ is the lift curve 


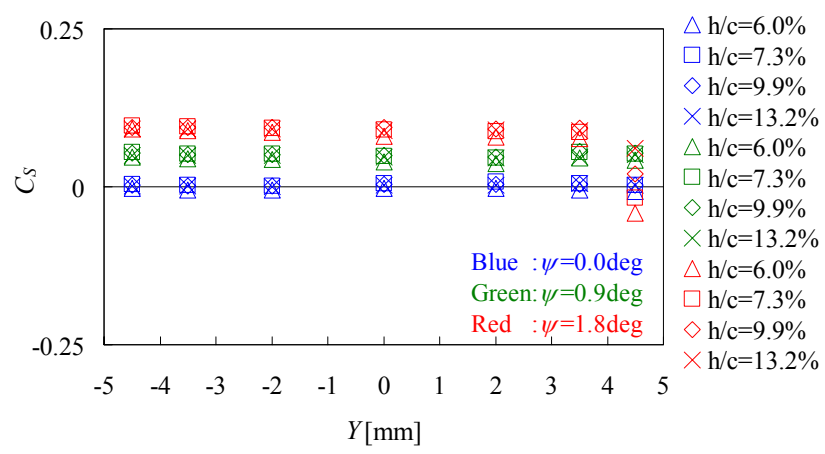

(a) rolling angle $\phi=2 \mathrm{deg}$

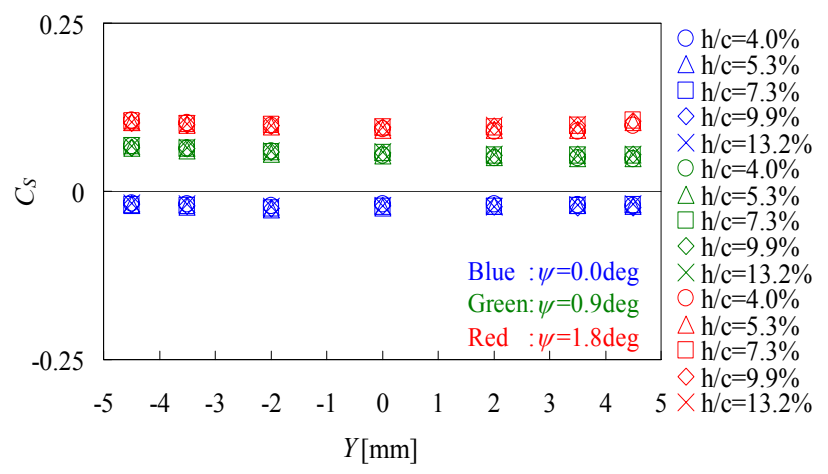

(b) rolling angle $\phi=0 \mathrm{deg}$

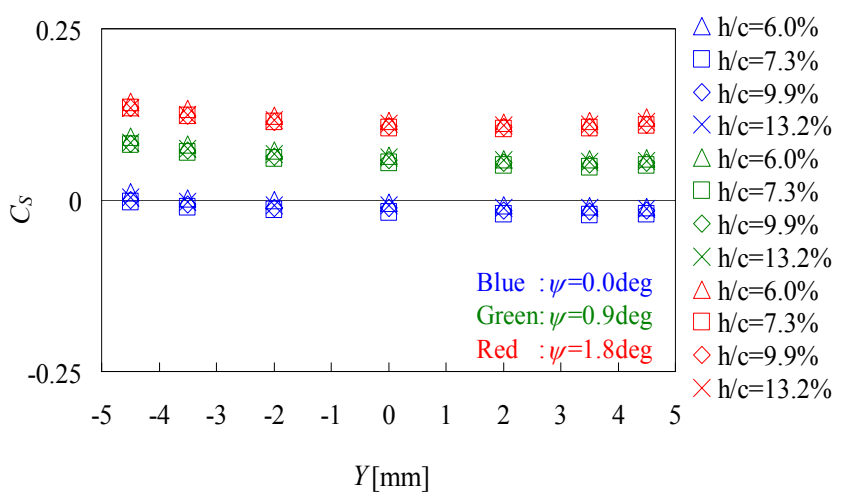

(c) rolling angle $\phi=-2 \mathrm{deg}$

Fig.4 Change of the side force coefficient by the lateral position

slope $d C_{S} / d \psi$ of the side wings. In this case, the lift curve slope $d C_{S} / d \psi=0.065$ on average. Moreover, because the change in $C_{S}$ by $h / c$ is small for any $Y$ position, the influence of $h / c$ to the side force is very small and thus can be ignored. Moreover, in yawing angle $\psi=0$ (cruise condition), $C_{S}$ is independent of the $Y$ position. Therefore, little stabilization effect from the ground effect of the side wings can be expected. According to the relationship of the rolling angle and the $C_{S}$ in position $Y=-4.5 \mathrm{~mm}$ at $\psi=0.9$ degrees and 1.8 degrees, the $C_{S}$ with a left rolling angle ( $\phi$ is positive) is larger than the $C_{S}$ with a right rolling angle ( $\phi$ is negative). As can be seen in Fig. 4(c), when the yaw is not zero and the $Y$ position is negative, repulsion from the side wall occurred. These two results are thought to be due to the combination of the following two phenomena. First, when the angle-of-attack of the side wings is high, then the air cushion effect appears. Second, when the distance between the tip of the side wings and a side wall becomes narrow due to the roll, then the effect works effectively. This is similar to the effect 


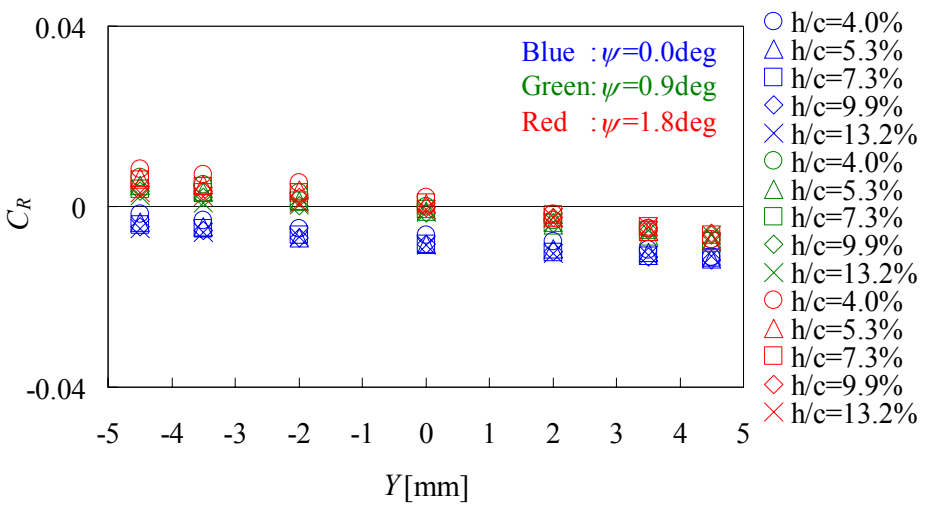

Fig. 5 Change of the rolling moment coefficient by the lateral position

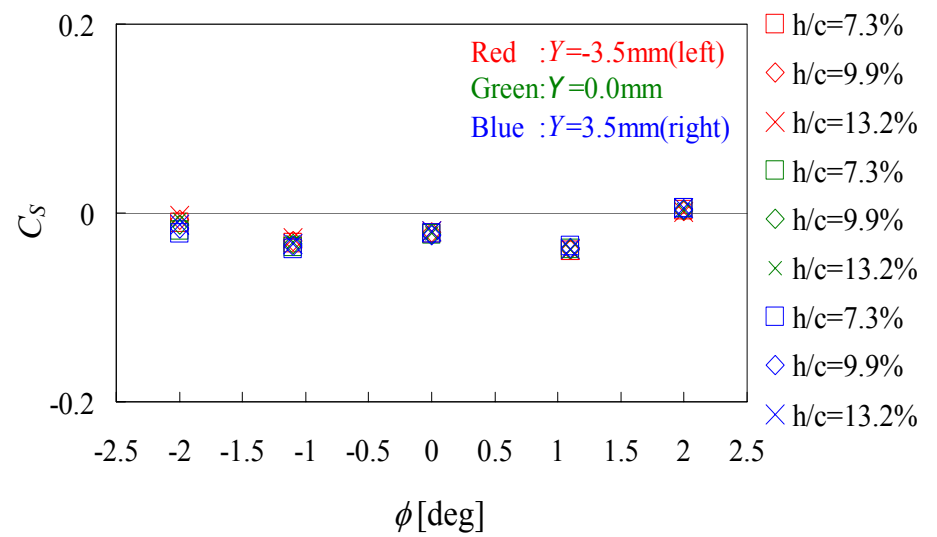

Fig.6 Change of the side force coefficient by the rolling angle

of an end plate to produce the air cushion effect because the gap between the tip of the side wing and the side wall becomes narrow due to the roll.

Change of the rolling moment coefficient $C_{R}$ by the lateral position inside the guide way $Y$ is shown in Fig. 5. This figure shows that $C_{R}$ decrease as $Y$ increases. This result means that the counterclockwise rolling moment increases when the body approaches the right-hand side wall and that the clockwise rolling moment increases when the body approaches the left-hand side wall. This result shows that the lift becomes large as the gap between the main wing and the side wall becomes small. This was expected from previous experimental results, and this phenomenon has also appeared in actual levitation runs as a rising of the wing when close to the side wall.

Change of the side force coefficient $C_{S}$ by the rolling angle $\phi$ is shown in Fig. 6. If repulsion from the side wall exists, then $C_{S}$ should become smaller as $\phi$ becomes larger due to the repulsion from the side wing approaching the side wall from the roll. However, the results in Fig. 6 do not show such a tendency. In the range of the rolling angles of -2 to +1 degree, $C_{S}$ is almost constant. Moreover, when the rolling angle $\phi$ is 2 degrees, then the tendency for suction by the side wall occurs in the opposite direction.

A summary of the above-mentioned results of our wind tunnel experiments is as follows.

1. Although the air cushion effect could be seen in the case of large angles of attack of the side wings, the effect was not so large. Therefore, a lateral restoring force by the ground effect of the side wing can not be reliably expected. 
2. In the yawing direction, it was completely unstable.

3. In the rolling direction, it was unstable or weakly stable.

According to the above results, there is a limit to the self-stabilization by the ground effect of the side wing. Therefore active control is necessary for a levitation run of the ART002 without surface contact.

\section{Levitating Run Experiment by the ART002}

\subsection{Outline of the Run Experiment for Model ART002}

The results of the wind tunnel experiment show that self-stability of the body front section in the guide way can not be expected. Considering this conclusion, a previously developed attitude control program was improved upon, and a test run experiment was conducted. The purpose of this improvement was to decide the control parameter using the control theory that incorporates wind tunnel experiment data. However, in this experiment, because the data are insufficient, the control parameter was not decided theoretically. This experiment was conducted in order to check the new control program and detect any problems related to the control equipment. The ART002 is the $2^{\text {nd }}$ generation levitating Aero-Train model. The front view, side view, and top view of the ART002 are shown in Fig. 7. The system for measurement and control is shown in Fig. 8. Most of the parts of the body are made from CFRP or GFRP, and the total weight of the body is about $420 \mathrm{~kg}$. The takeoff speed is about $105 \mathrm{~km} / \mathrm{h}$ (about $30 \mathrm{~m} / \mathrm{s}$ ). The airfoil of the main wings is NACA6412 modified, the setting angle of the front wing is 4.8 degrees, and the setting angle of the rear wing is 2.8 degrees. The reason for this difference in setting angle is because if both wings have the same setting angle, then the lift of the rear wing becomes too large and balance cannot be maintained. More specifically, because the wake of the propeller tilts downward by the fairing of the propulsive motor pylon and the wake hits the rear main wing, the lift of the rear main wing increases. The position of center-of-gravity can be adjusted by changing the position of the batteries for the propulsive motors, control, and measurement. The
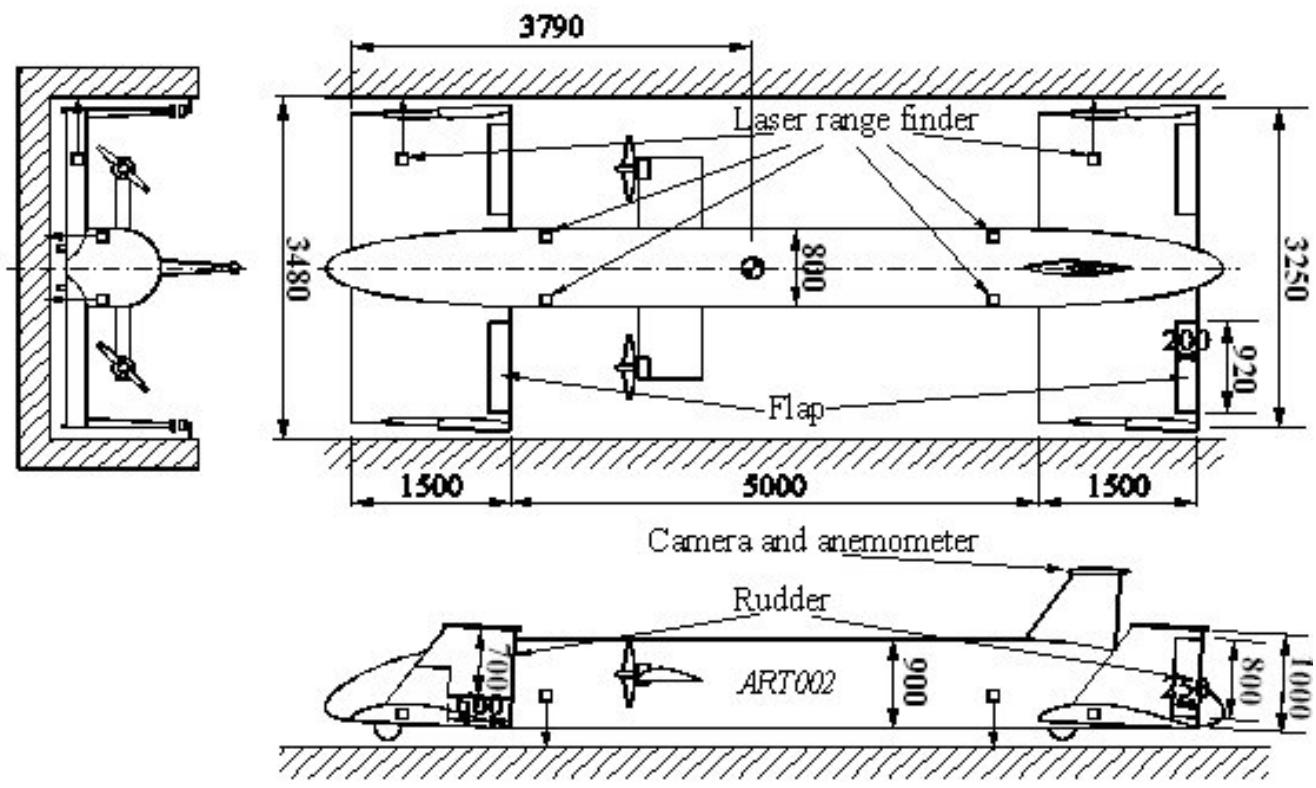

Fig.7 Experimental model of ART002 


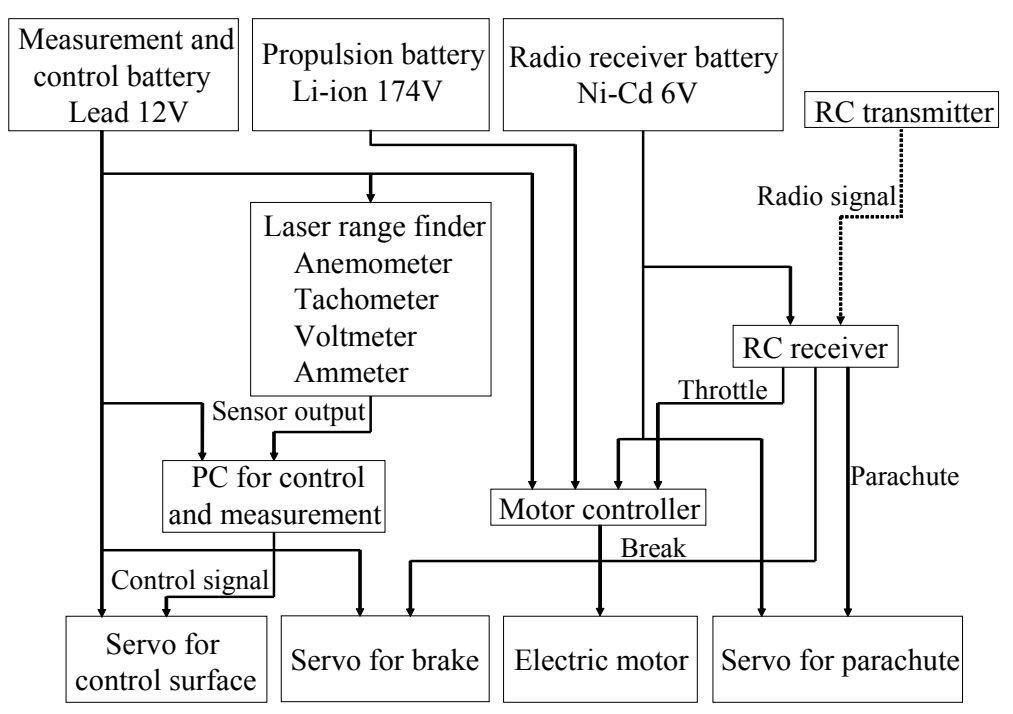

Fig.8 Schematic figure of the system of measurement and control

vertical stability becomes weak when the position of center-of-gravity is located too far back, and when it is too forward, the Aero-Train cannot levitate due to the rear and front lift balance. Therefore, in this test run, the center of gravity was set at $3790 \mathrm{~mm}$ from the leading edge of the front main wing. Propulsive force was generated by two propellers with a diameter of $640 \mathrm{~mm}$ attached on both sides of the body. Two DC electric motors drove these propellers. The battery for the propulsive motors was a Li-ion battery for electric vehicles, with a normal-rated voltage of $175 \mathrm{~V}$ and rated current of $12.8 \mathrm{Ah}$. The body positions and the angles inside the guide way were measured by six laser range finders, of which four were installed in the lower part of the fuselage, while the remaining two were installed in the front and rear right wings. The PC used for control and measurement acquired the output data from the laser range finders, anemometer, etc., and calculated the angle of each control surface, sending a control signal to each servomotor. The control surfaces were flaperons at the trailing edges of the main wings and rudders at the trailing edge of the side wings. Each control surface operated independently except for the rudder of the rear side wing. The power supply of the PC, the measuring instrument, and the servomotor was a $12 \mathrm{~V}$ lead battery commonly used in cars. The throttle, parachute, and brakes were manually controlled by radio from a car running behind the Aero-Train for safety.

The guide way was previously used by RTRI as a test track for a Maglev, and it was subsequently improved as the Flow Environment Simulator (towing wind tunnel). In this study, the length of the guide way used was $1900 \mathrm{~m}$. The section from $910 \mathrm{~m}$ to $1400 \mathrm{~m}$ from the start point was a section with a roof made from FRP. The inner width of the guide way was $3480 \mathrm{~mm}$. An electrical power cable, used as a device to prevent the body from jumping out, was also attached to the top edge of the side wall.

\subsection{Control Program}

The previous control program preserves the position of each laser range finder. Therefore, a certain control surface can be controlled only by the output of the nearest sensor. In order to control more effectively, we changed this control method into a system that first calculates the angles and the positions of the Aero-Train inside the guide way from the output of six laser range finders, then calculates their respective differential and 
integration values, and finally calculates the angle of each control surface by multiplying the gain by these values. This method can be expressed as follows.

$$
\left(\left(\begin{array}{c}
\delta_{R F} \\
\delta_{L F} \\
\delta_{R B} \\
\delta_{L B} \\
\delta_{F G} \\
\delta_{B G}
\end{array}\right)=\mathbf{P}\left(\begin{array}{c}
h \\
\theta \\
\phi \\
\psi \\
Y
\end{array}\right)+\mathbf{D} \cdot \frac{d}{d t}\left(\begin{array}{c}
h \\
\theta \\
\phi \\
\psi \\
Y
\end{array}\right)+\mathbf{I} \cdot \int\left(\begin{array}{c}
h \\
\theta \\
\phi \\
\psi \\
Y
\end{array}\right) d t\right.
$$

$\delta_{R F}$ : front right flaperon angle, $\delta_{L F}$ : front left flaperon angle,

$\delta_{R B}$ : rear right flaperon angle, $\delta_{L B}$ : rear left flaperon angle,

$\delta_{F G}$ : front rudder angle, $\delta_{B G}$ : rear rudder angle,

$h:$ difference of surfacing height and setting height $[\mathrm{m}]$,

$\theta$ : pitching angle [deg], $\phi$ : rolling angle [deg], $\psi:$ yawing angle [deg]

$Y$ : lateral position in guide way

The gain matrices P, D, and I are determined from the former PID controller data, as referenced in the beginning, and are adjusted by repeating the experiments. Gain values larger than those of the previous PID controller are applied to unstable yawing and rolling angles. In particular, large gain values are applied to the differential values, while small gain values are applied to the stable pitching angle. For safety, when the pitching angle becomes more than +1 degree, the flaperon of each main wing is raised and the body is grounded. The control flow chart is shown in Fig. 9. When the airspeed exceeds $25 \mathrm{~m} / \mathrm{s}$, then the control shifts from the acceleration phase to the attitude control phase. The control cycle is $20 \mathrm{~ms}(50 \mathrm{~Hz})$, and a moving average in five points is adopted in the calculation of the differential value for noise reduction.

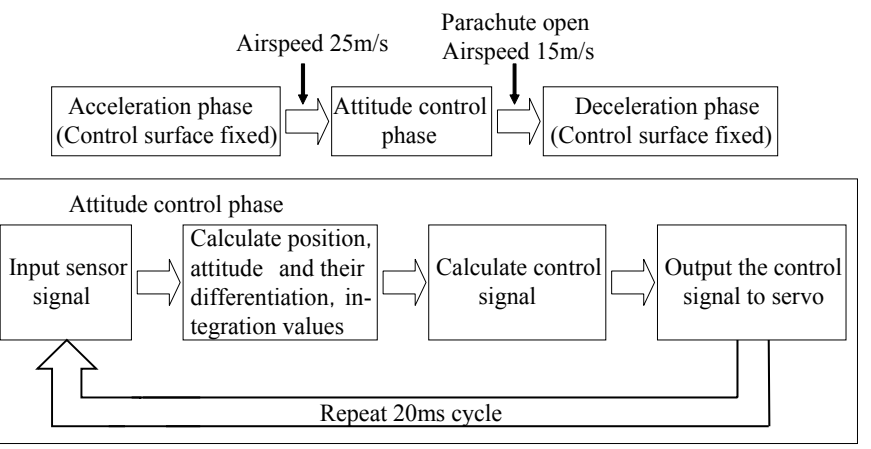

Fig.9 Flowchart of control system

\subsection{Results of Levitating Run and Discussion}

The run experiment was repeated while adjusting the gain matrix. The time series data of the run tests are shown in Fig. 10(a) - (f). As comparative data, the data of the former PID controller are shown in the same figure. The "before" line indicates previous data, and the "after" line indicates the current data. This graph shows the data for 10 seconds after a certain time interval after levitation, which is different for the "before" and "after" data.

Let us first examine Fig. 10(a), showing the levitation height $h$, and Fig. 10(b), showing the pitching angle $\theta$. The levitation height is the average value of the four laser range finders in the body, and it is set to 0 when the body is on the ground. Figure 10(a) indicates 
that the body levitates at about $0.06 \mathrm{~m}$ in both of the "before" and "after" data. In Fig. 10(b), the pitching angle $\theta$ changes in the range between -0.8 degree and 0.2 degree, and the Aero-Train flies slightly pitched down. The increase in the lift of the rear main wing due to propeller wake may be the cause of this phenomenon. The levitation height and the pitching angle are not stable because these two quantities are sensitive to speed and because they are influenced by disturbance due to natural winds (especially, crosswinds) and throttle control, etc. However, this state is sufficient to evaluate the stability inside the guide way in a levitating run because the front wheels touch the ground neither in the "before" nor the "after" case. The rear wheels are lifted off of the ground when the front wheels are lifted off of the ground because the pitching angle is negative.

Next, the quantities related to the stability of the lateral direction inside the guide way are investigated in Fig. 10(c), (d), and (e). The rolling angle and the lateral position oscillate at about $0.7 \mathrm{~Hz}$ in the "before" data. Moreover, the oscillation of the yawing angle is at the same frequency. These results show the meandering movement inside the guide way. A

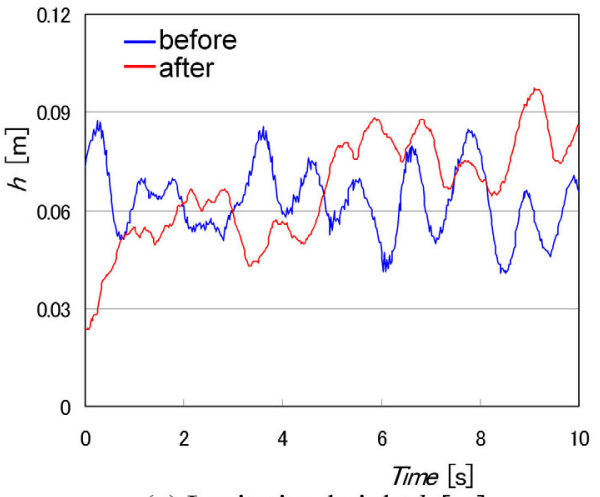

(a) Levitation height $h[\mathrm{~m}]$

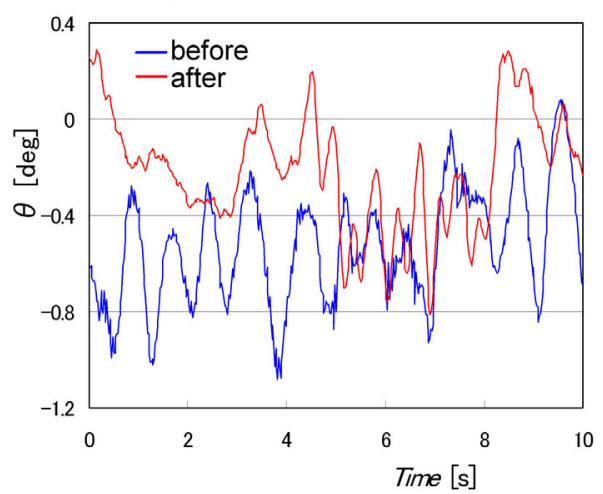

(b) Pitching angle $\theta[\mathrm{deg}]$

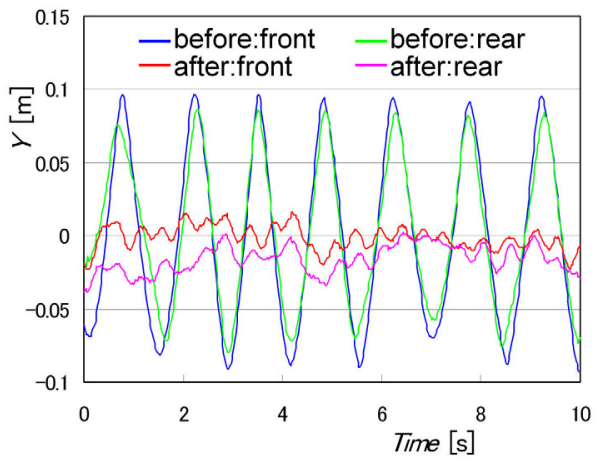

(c) Lateral positon $Y[\mathrm{~m}]$

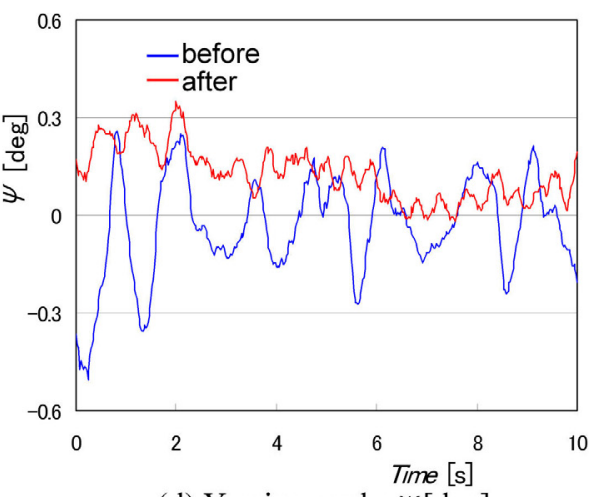

(d) Yawing angle $\psi[\mathrm{deg}]$

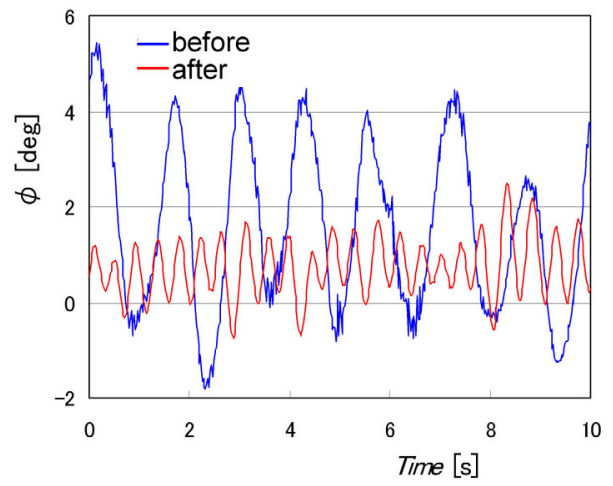

(e) Rolling angle $\phi[\mathrm{deg}]$

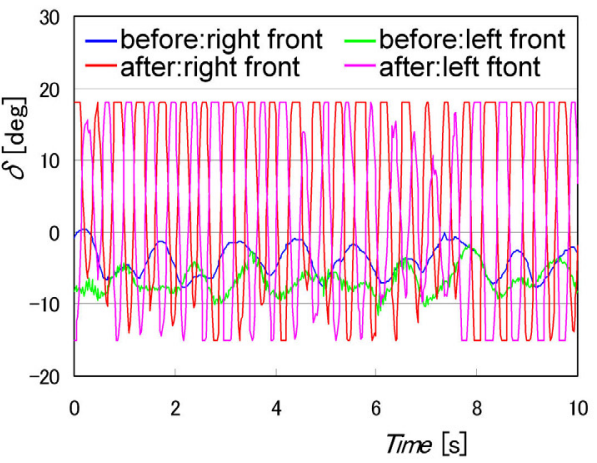

(f) Flaperon angle $\delta$ [deg]

Fig.10 Change of the data by the control parameters 
large oscillation is not seen in either the results of the yawing angle or the lateral position in the "after" data. The body might be flying without contacting the side wall and is almost steady. The amplitude of the roll angle oscillation was reduced by more than $50 \%$ compared to that in the "before" case. However, the oscillation of about $3 \mathrm{~Hz}$ between 0 degree and 1.8 degrees still remains. In order to search for the cause of this oscillation, the angles of the flaperons of the front main wings are shown in Fig. 10(e). Compared with the "before" case, the right flaperon and left flaperon move in opposite directions, and the amplitude of the motion is very large in the "after" case. The opposing oscillation of the right and left flaperons is due to the gains of the rolling angle and the rolling speed. Although the flaperon angle is restricted to a range of -15 to 18 degrees from mechanical restriction, the movable range is used up by the rolling control in the "after" case. Considering the fact that the pitch and the levitation height must be controlled in addition to the roll, this is unfavorable. The following are thought to be possible causes.

1. Delay of the servomotors

2. Delay of the rolling speed by moving average processing

3. Insufficiency of gain value adjustment

Because the ART002 is long and slender, its moment of inertia around the rolling axis is extremely small compared with the moment of inertia around the other axes. Therefore, it is easy for it to rotate in the rolling direction, and the speed of roll is also fast. The following two modifications will improve the rolling problem with respect to the time delay. First, the drive motor of the control surface can be changed into a high-speed and high-response servomotor. Second, a modification can be made to directly detect the angular velocity using gyroscope sensors, etc. The rolling control uses up the movable range of the flaperon. The reason for this is thought to be because the gain is too large and needs to be adjusted more. If the rolling oscillation does not become small after adjusting the gain, or if the rolling control uses up the movable range of the flaperon, then it is necessary to increase the flaperon area in addition to modifying the delay of the control system. Moreover, if the front part of the body is improved based on the results of these wind tunnel tests, the body can become more stabilized in the lateral direction.

\section{Conclusions}

Wind tunnel experiments of the static stability of the front body section of the ART002 were performed. Based on the results, we can conclude that the control program of the ART002 was improved. Subsequently, a levitation test run of the ART002 was conducted. The results are summarized as follows.

1. Although the air cushion effect is seen in the case of large angles of attack of the side wings, the effect was not large. Therefore, stability in the lateral direction by the ground effect of the front side wing should not be expected. Moreover, the restoring force by the ground effect should also not be expected. The front section of the body is unstable or is weakly stable in the direction of yawing and rolling. From these results, it can be concluded that active control is necessary for a levitating run of the ART002 without surface contact.

2. In the levitating test run of the ART002, levitated running without surface contact was achieved inside the guide way. However, the vibration of the rolling remained. It is thought that the delay of the control system and the insufficiency of the gain adjustment are the causes of this vibration.

The Aero-Train is controlled by active control when in a levitation run, and this has been stabilized in the present experiment. For the future third generation model, i.e., the 
ART003, it will be necessary to improve static stability in order to achieve levitation runs without surface contact by extending the body, forward swept side wing, and forward swept end plate of the side wing while reducing the distance between the side wing and the side wall by extending the wingspan of the main wing.

\section{Acknowledgements}

The authors thank the juridical foundation "Railway Technical Research Institute," which lent us the guide way free of charge, and Mr. Yasunori Matsushima of Matsushima Densetsu Kogyo Ltd. for his help in the test runs.

\section{References}

(1) Ollia, R. G., Histrical Reverw of WIG Vehicles, J. Hydronautis, 14-3, (1980), pp.65-76.

(2) Ippisch, A. M., Der 'Aerodynamische Bodeneffekt' und die Entwicklung des Flugfl chen-(Aerofoil)-Bootes, Luftfahrttechnik Raumfahrttechnik, 10 , (1964), Nr.10, Oktober, pp.261-269.

(3) Nangia, R. K., Aerodynamic and Hydrodynamic Aspects of High Speed Water Surface Craft, Aeronautical Journal, June/July, (1987), pp.241-268.

(4) Jorg, G. W., History and Development of the 'Aerodynamic Ground Effect Craft'(AGEC) with Tandem Wings, Symp. Proc. Ram Wing and Ground-Effect Craft, Royal Aeronautical Society, (1987), pp.87-109.

(5) Tomaru, H. and Kohama, Y., Wind-Tunnel Investigation of Aerofoil for Wing in Ground Effect, Journal of Japan Society of Fluid Mechanics, (in Japanese), Vol. 10, (1991), pp.47-60.

(6) Tomaru, H. and Kohama, Y., Aerodynamics of Tandem-WIG in Guide Way, Journal of Japan Society of Fluid Mechanics, Vol.11, (1992), pp.45-52.

(7) Kohama, Y. et al., Experimental and Numerical Study of Aerodynamic Characteristics of a Ground Effect Transport System (GETS), Journal of the Japan Society for Aeronautical and Space Sciences, (in Japanese), Vol. 47, (1999), pp.79-87.

(8) Ahamed. M. R. and Kohama. Y., Experimental Investigation on the Aerodynamic Characteristics of a Tandem Wing Configuration in Close Ground Proximity, JSME International Journal, Vol.42, No.4, (1999), pp.612-618.

(9) Kohama, Y. et al., Flight Dynamics and Development of the Stability Control Method of the Aero-Train (1st Report, Flight Test by Pushing), Transactions of the Japan Society of Mechanical Engineers, Series B, (in Japanese), Vol.68, No.665, (2002), pp.102-107

(10) Watanabe, H. et al., Improvement in Performance of Aerotrain by Controlling Side-Wing/Wing Interaction, Transactions of the Japan Society of Mechanical Engineers, Series B, (in Japanese), Vol.69, No.686, (2003), pp.2230-2236.

(11) Yoon, D. et al., Improvement of the Aerotrain Aerodynamic Performance by Controlling Wing Interactions, Transactions of the Japan Society of Mechanical Engineers, Series B, (in Japanese), Vol.70, No.694, (2004), pp.1433-1440.

(12) Ito, H. et al., The Design and Performance of the Low-Turbulence Wind Tunnel, Tohoku University, Mem. Inst. High Speed Mech, Tohoku Univ., (in Japanese), 44(1980), pp.93 151. 